TRANSACTIONS OF THE

AMERICAN MATHEMATICAL SOCIETY

Volume 280. Number 1, November 1983

\title{
THE WORD PROBLEM FOR LATTICE-ORDER GROUPS
}

BY

\author{
A. M. W. GLASS AND YURI GUREVICH ${ }^{1}$ \\ To. W. W. Boone on the occasion of his 60 th birthday
}

\begin{abstract}
.
THEOREM. There is a finitely generated one relator lattice-ordered group with insoluble (group) word problem.
\end{abstract}

We prove

THEOREM. There exists a finitely presented lattice-ordered group with insoluble word problem.

COROLLARY. There is a one relator finitely generated lattice-ordered group with insoluble word problem.

The Theorem is the obvious analogue of the corresponding result for groups. However, the Corollary has no analogue for groups since any one relator finitely generated group has soluble word problem [7].

The proofs we will give are completely self-contained except for two well-known results from recursion theory (I and II below).

0. Introduction. A lattice-ordered group is a group and a lattice such that the group operation distributes over the lattice operations. An example (which we will need later) is $\mathbf{A}(\mathbf{R})=\operatorname{Aut}(\langle\mathbf{R}, \leqslant\rangle)$, the order-preserving permutations of the real line, $\mathbf{R}$; the group operation is composition, and the lattice operations are the pointwise ones (i.e., if $f, g \in \mathbf{A}(\mathbf{R})$, then $f g, f \vee g$ and $f \wedge g$ are the order-preserving permutations of $\mathbf{R}$ defined by: $\alpha(f g)=(\alpha f) g, \alpha(f \vee g)=\max \{\alpha f, \alpha g\}$ and $\alpha(f \wedge g)=\min \{\alpha f, \alpha g\}(\alpha \in \mathbf{R})$, respectively).

A presentation of a lattice-ordered group with generators $x_{i}(i \in I)$, and relations $r_{j}(\mathbf{x})=e(j \in J)$ can be realised by taking the quotient of the free lattice-ordered group $F$ on $\left\{x_{i}: i \in I\right\}$ by the normal convex sublattice subgroup generated by the elements $\left\{r_{j}(\mathbf{x}): j \in J\right\}$ of $F$. If $I$ and $J$ are both finite we will say that the lattice-ordered group is finitely presented. Note that the $r_{j}(\mathbf{x})$ are formed from $\mathbf{x}$ by using possibly both the lattice and group operations.

Received by the editors February 13, 1981 and, in revised form, May 20, 1982.

1980 Mathematics Subject Classification. Primary 03D40, 06F15, 20F10; Secondary 20 B07.

'This research was begun in Summer 1977 when the first author was visiting the University of Alberta, and completed in Spring 1980 when the second author was visiting Bowling Green State University. The authors gratefully acknowledge the friendliness and warm hospitality they received at the respective universities. 
We will obtain a certain primitive recursive set $R$ of relations, involving only a finite set of letters correlated with elements of $\mathbf{A}(\mathbf{R})$, such that the subset $S$ of $R$ comprising those members of $R$ which hold in $\mathbf{A}(\mathbf{R})$ is nonrecursive. We will then show that there is a finite set $S_{0}$ of relations which hold in $\mathbf{A}(\mathbf{R})$ and imply $S . S_{0}$ will be constructed from a finite set $X_{0}$ of letters, consisting of the letters which appear in $R$ and new elements correlated with additional elements of $\mathbf{A}(\mathbf{R})$. A member of $R$ will then be implied by $S_{0}$ if and only if it belongs to $S$. Hence the finitely presented lattice-ordered group $\left(X_{0} ; S_{0}\right)$ will have insoluble word problem; i.e., the word problem for $\left(X_{0} ; S_{0}\right)$ is not recursively soluble. Roughly speaking, $S_{0}$ corresponds to the characteristic function of a recursively enumerable set that is not recursive. Indeed, the representation of each recursive function by a finite set of relations is the main step in our proof-see $\S 4$.

The method we use in our proof for coding in recursive functions comes from [9] where a similar technique was used to give an elementary proof of the existence of a finitely presented group with insoluble word problem. The intuition gained from reading McKenzie and Thompson's paper has proved invaluable to us. We are most grateful to Professor W. W. Boone for making us aware of it. ${ }^{2}$

Caution. [9] makes essential use of an element $R$ of order 3 (which juggles the levels). Since lattice-ordered groups have no torsion elements, we have had to develop a totally different strategy to prove our theorem. This has meant we have had to originate new results in ordered permutation groups concerning conjugating several elements simultaneously by the same conjugator (see Appendix for proofs). This new technique is necessitated by the failure of the amalgamation property [2, Theorem 10C] and is an essential departure from [9]. We do not know if the standard finitely presented groups with insoluble word problem (see, e.g.. [0] and [1]) can be made into lattice-ordered groups - they are torsion-free. Also, we do not know whether the lattice-ordered groups (as opposed to groups) given by these finite presentations have insoluble word problem - the lack of a unique normal form and the absence of the amalgamation property make this a difficult problem.

The only results on word problems for lattice-ordered groups to date have concerned free objects in certain varieties of lattice-ordered groups [5], [6] and [8] and the existence of a recursively presented lattice-ordered group with insoluble word problem [3].

We are most grateful to the referees, especially George McNulty, for their careful reading of the manuscripts. Their attention to detail has led to considerable amplification in many places we had left obscure. The readability of the paper (such as it is) owes a great deal to them.

1. Background. We will use $\omega$ for the set of nonnegative integers, $\mathbf{Z}$ for the set of integers, and $\mathbf{Z}^{+}$for the set of positive integers. ${ }^{\omega} \omega$ will be used for the set of functions from $\omega$ into $\omega$. In contrast to our notation for permutations, we will write

\footnotetext{
${ }^{2}$ After this article was written, we learned from Ralph N. McKenzie that Richard J. Thompson had independently solved the problem but had not published it.
} 
elements of ${ }^{\omega} \omega$ on the left; so, if $f, g \in{ }^{\omega} \omega, f g$ will be that member of ${ }^{\omega} \omega$ defined by $f g(n)=f(g(n))(n \in \omega)$.

Before embarking on the proof of the Theorem, we develop the notation and basic facts that we will need about lattice-ordered groups and $\mathbf{A}(\mathbf{R})$.

LeMma 0 . Let $G$ be any lattice-ordered group and $x, y, z \in G$.

(i) If $x \wedge y=e$, then $x^{-1} y x=y$.

(ii) If $x(x \wedge y)^{-1} \wedge y=e$ and $e \leqslant z \leqslant y$, then $(x \wedge y)^{-1} z(x \wedge y)=x^{-1} z x$.

(iii) If $x \wedge y x^{-1}=e, e \leqslant z \leqslant y \& y z=z y$, then $x \leqslant y \& x z=z x$.

Proof. Since $(x \wedge y)^{-1}=x^{-1} \vee y^{-1}$, we have $x(x \wedge y)^{-1} y=y \vee x$ for all $x, y \in G$.

(i) If $x \wedge y=e$, then $x y=y \vee x=x \vee y=y x$.

(ii) If $x(x \wedge y)^{-1} \wedge y=e \& e \leqslant z \leqslant y$, then $x(x \wedge y)^{-1} \wedge z=e$. By (i), $x(x \wedge y)^{-1} z=z x(x \wedge y)^{-1}$. Thus (ii) holds.

(iii) If $x \wedge y x^{-1}=e$, then $y x^{-1} \geqslant e$ (so $\left.y \geqslant x\right)$ and $x y x^{-1}=y x^{-1} x=y$ by (i). Since $z \geqslant e, x \wedge z \wedge y x^{-1}=e$; hence $y x^{-1}(x \wedge z)=(x \wedge z) y x^{-1}=y(x \wedge z) x^{-1}$ ( $y$ commutes with $x \& z)$. Thus $x \wedge z=x(x \wedge z) x^{-1}=x \wedge x z x^{-1}$. As $x, z \leqslant y$, $x \wedge(x \vee z) x^{-1}=e$. Therefore $x \vee z=(x \vee z) x^{-1} x=x(x \vee z) x^{-1}$ by (i). Consequently, $x \wedge z=x \wedge x z x^{-1} \& x \vee z=x \vee x z x^{-1}$. Since lattice-ordered groups are distributive lattices, $z=x z x^{-1}$, proving (iii).

For $g \in \mathbf{A}(\mathbf{R})$, let $\operatorname{supp}(g)=\{\alpha \in \mathbf{R}: \alpha g \neq \alpha\}$. If $\operatorname{supp}(g)$ is precisely one interval of $\mathbf{R}$, we will say that $g$ has one bump; if $\operatorname{supp}(g)$ is bounded in $\mathbf{R}$, we will say that $g$ is bounded.

Observe that if $e<f, g \in \mathbf{A}(\mathbf{R})$, then $f \wedge g=e$ if and only if $\operatorname{supp}(f) \cap \operatorname{supp}(g)$ $=\varnothing$. Also, $\operatorname{supp}\left(h^{-1} g h\right)=[\operatorname{supp}(g)] h$. Hence if $g$ is bounded and has one bump and $h>e$, then $h^{-1} g h \wedge g=e$ if and only if $h$ maps the interval of support of $g$ completely to the right of the interval of support of $g$. Further, if $f, f_{1}>e$, then $f_{1} \wedge f f_{1}^{-1}=e$ if and only if $\alpha f_{1}=\alpha f$ for all $\alpha \in \operatorname{supp}\left(f_{1}\right)$. In this case, $\operatorname{supp}\left(f_{1}\right) \subseteq \operatorname{supp}(f)$ and $f=f_{1} \vee f f_{1}^{-1}$. All of these facts are easily verified and are standard fare in the study of ordered permutation groups (see $\$ \S 1.9$ and 2.1 of [2]).

\section{Interpretation in $\mathbf{A}(\mathbf{R})$.}

We now construct elements of $\mathbf{A}(\mathbf{R})$ which will realise all the relations we require later. We present the interpretation first since it led us to the eventual finitely presented lattice-ordered group with insoluble word problem. Indeed, it is the picture that led to the construction of such a lattice-ordered group.

In $\mathbf{A}(\mathbf{R})$, there are elements $a_{0}, b_{0}, c_{0}>e$ each bounded and having one bump, such that:

(a) $a_{0} \wedge b_{0}^{-n} a_{0} b_{0}^{n}=e=b_{0} \wedge c_{0}^{-n} b_{0} c_{0}^{n}$ for all $n \in \mathbf{Z} \backslash\{0\}$,

(b) $b_{0}^{-m} a_{0} b_{0}^{m}<b_{0}$ and $c_{0}^{-m} b_{0} c_{0}^{m}<c_{0}$ for all $m \in \mathbf{Z}$,

(c) between $\operatorname{supp}\left(b_{0}^{-m} a_{0} b_{0}^{m}\right)$ and $\operatorname{supp}\left(b_{0}^{-n} a_{0} b_{0}^{n}\right)$ there is a nondegenerate interval of $\mathbf{R}$ whenever $m$ and $n$ are distinct integers, and

(d) similarly for $\operatorname{supp}\left(c_{0}^{-m} b_{0} c_{0}^{m}\right)$ and $\operatorname{supp}\left(c_{0}^{-n} b_{0} c_{0}^{n}\right)$. 
130

A. M. W. GLASS AND YURI GUREVICH

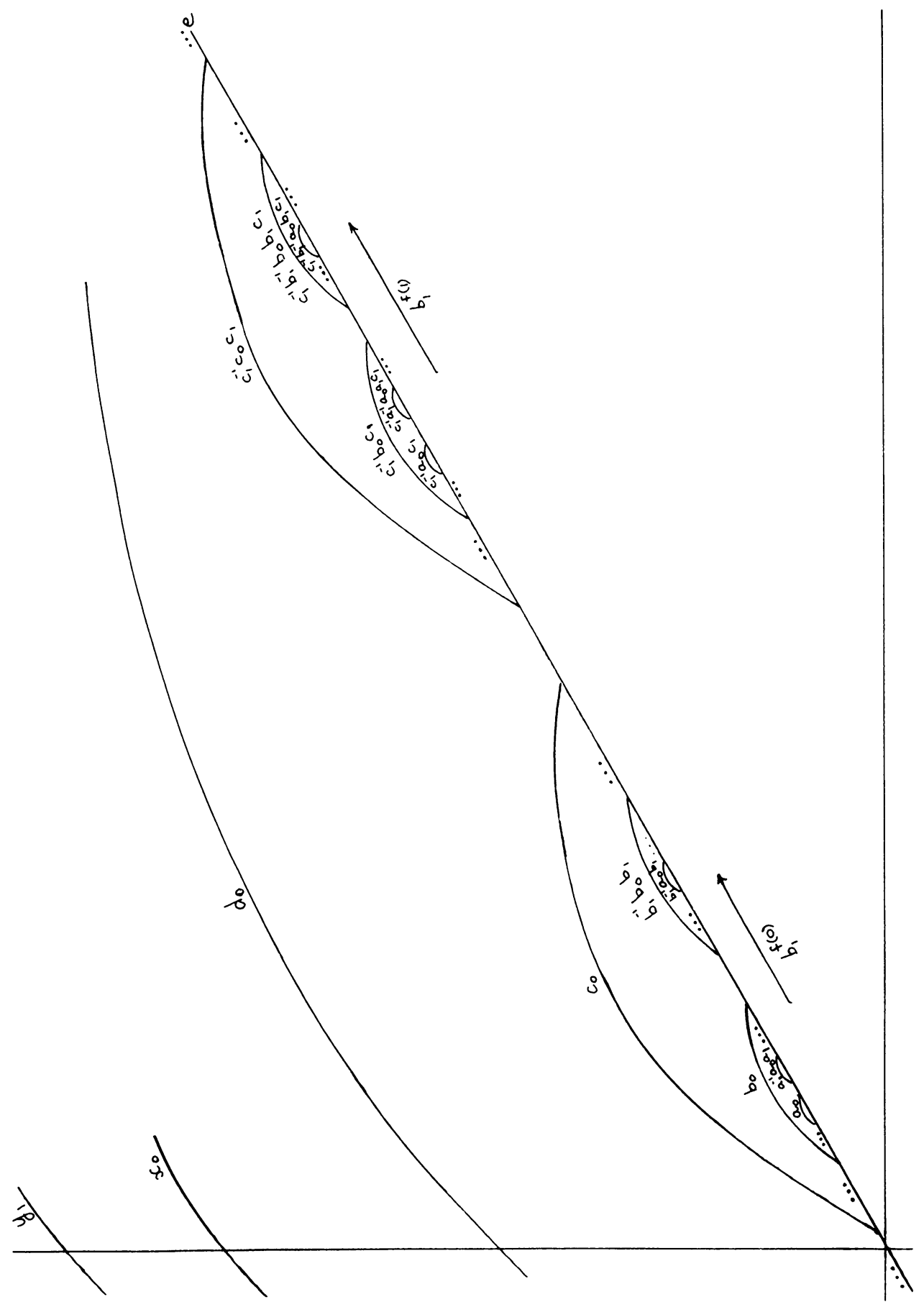


The picture above describes the situation-by (a) and the remarks in $\S 1$, the support of $b_{0}^{-n} a_{0} b_{0}^{n}$ is indeed to the left of the support of $b_{0}^{-m} a_{0} b_{0}^{m}$ if $m>n$. So we can satisfy (c), (a) and (b) simultaneously; similarly also (d).

Because of the bump structure, there is $a_{3} \in \mathbf{A}(\mathbf{R})$ such that $a_{3}^{-1} a_{0} a_{3}=b_{0}$ and $a_{3}^{-1} b_{0} a_{3}=c_{0}$. Since the actual definition of $a_{3}$ (as opposed to these vital properties) is immaterial, we omit the details here - they can be found in the Appendix. Let $d_{0}=a_{3}^{-1} c_{0} a_{3}, x_{0}=a_{3}^{-1} d_{0} a_{3}$ and $y_{n}=a_{3}^{-n} x_{0} a_{3}^{n}\left(n \in \mathbf{Z}^{+}\right)$. Let $W=\left\{y_{1}^{n_{1}} \cdots y_{k}^{n_{k}}\right.$ : $\left.k \in \mathbf{Z}^{+}, n_{1}, \ldots, n_{k} \in \mathbf{Z}\right\}$ and write $x * y$ for $y^{-1} x y(x * y z$ is shorthand for $x *(y z))$. By (a), (b), the remarks in $\S 1$ and the definition of $a_{3},\left\{x_{0} * w: w \in W\right\}$ forms a pairwise disjoint set. Let $d_{1} \in \mathbf{A}(\mathbf{R})$ be the pointwise supremum of it. (Note that $x_{0} \wedge d_{1} x_{0}^{-1}=e$, i.e., $a_{3}^{-1} d_{0} a_{3} \wedge d_{1}\left(a_{3}^{-1} d_{0} a_{3}\right)^{-1}=e$-so $x_{0} \vee d_{1} x_{0}^{-1}=d_{1}$.) Let $c_{1} \in$ $\mathbf{A}(\mathbf{R})$ be the pointwise supremum of the pairwise disjoint set of elements $\left\{d_{0} * d_{1}^{n} w\right.$ : $n \in \mathbf{Z}, w \in W\}, b_{1} \in \mathbf{A}(\mathbf{R})$ be the pointwise supremum of the pairwise disjoint set of elements $\left\{c_{0} * c_{1}^{m} d_{1}^{n} w: m, n \in \mathbf{Z}, w \in W\right\}$, and $a_{1} \in \mathbf{A}(\mathbf{R})$ be the pointwise supremum of the pairwise disjoint set $\left\{b_{0} * b_{1}^{m} c_{1}^{n} d_{1}^{k} w: m, n, k \in \mathbf{Z}, w \in W\right\}$. By construction $a_{1}, b_{1}, c_{1}$ and $d_{1}$ each commute with each other, $a_{3}^{-1} c_{1} a_{3}=d_{1}, a_{3}^{-1} b_{1} a_{3}$ $=c_{1}$ and $a_{3}^{-1} a_{1} a_{3}=b_{1}$. Also, since $a_{1} \uparrow \operatorname{supp}\left(b_{0}\right)=b_{0} \uparrow \operatorname{supp}\left(b_{0}\right), a_{1} b_{0}=b_{0} a_{1}$. Let $a_{2}$ be the pointwise supremum of the pairwise disjoint set $\left\{a_{0} * a_{1}^{n}: n \in \mathbf{Z}^{+}\right\}$. So $a_{0} \wedge a_{2}=e$ and $a_{1}^{-1} a_{2} a_{1}<a_{2}$. Let $a_{6}$ and $a_{7}$ be the pointwise suprema of the pairwise disjoint sets $\left\{a_{0} * c_{1}^{m}: m \in \omega\right\}$ and $\left\{a_{0} * c_{1}^{-m}: m \in \mathbf{Z}^{+}\right\}$. Note that $a_{6} \wedge a_{7}$ $=e, c_{1}^{-1}\left(a_{6} \vee a_{7}\right) c_{1}=a_{6} \vee a_{7}, a_{0} \wedge a_{6} a_{0}^{-1}=e, c_{1}^{-1} a_{6} c_{1} \leqslant a_{6}, \quad c_{1} a_{0} c_{1}^{-1} \leqslant a_{7}$ and $c_{1} a_{7} c_{1}^{-1} \leqslant a_{7}$.

Because of bump structure, we can find $a_{4}, a_{5} \in \mathbf{A}(\mathbf{R})$ such that $a_{4}^{-1} a_{0} a_{4}=a_{0}$, $a_{4}^{-1} a_{1} a_{4}=b_{1}, \quad a_{4}^{-1} b_{1} a_{4}=c_{1}$ and $a_{4}^{-1} c_{1} a_{4}=d_{1} ; \quad a_{5}^{-1} a_{0} a_{5}=a_{0}, a_{5}^{-1} b_{0} a_{5}=c_{0}$ and $a_{5}^{-1} c_{1} a_{5}=c_{1}$-see the Appendix for the details. Hence $a_{0} * a_{1}^{m} a_{5}=a_{0} * b_{1}^{m}$ for all $m \in \mathbf{Z}$.

We have coded in five "levels" of $\mathbf{Z}$ via $a_{0}, a_{1}, b_{1}, c_{1}$ and $d_{1}$, e.g., $a_{0} a_{1}^{n} b_{1}^{m}$ will code in 1 at the lowest level, $n$ at the next level, $m$ at the third level, and 0 at the two highest levels. The purpose of $a_{3}, a_{4}$ and $a_{5}$ is to help us to pass from one level to another. This wll be vital in the proof of Lemma 2 when coding in the composition of two functions belonging to ${ }^{\omega} \omega$ (see $\S 4$ ). Since the class of lattice-ordered groups fails to satisfy the amalgamation property, the existence of such $a_{3}, a_{4}$ and $a_{5}$ is by no means guaranteed. The construction of $a_{0}, a_{1}, b_{2}, c_{1}$ and $d_{1}$ has been carefully contrived to ensure that there are indeed elements $a_{3}, a_{4}$ and $a_{5}$ having the desired properties.

We now code $f \in{ }^{\omega} \omega$ into $\mathbf{A}(\mathbf{R})$ via $a_{f}$ where

$$
\operatorname{supp}\left(a_{f}\right)=\cup\left\{\operatorname{supp}\left(c_{0} * c_{1}^{m} d_{1}^{n}\right): m \in \omega, n \in \mathbf{Z}\right\}
$$

and

$$
a_{f} \uparrow \operatorname{supp}\left(c_{0} * c_{1}^{m} d_{1}^{n}\right)=b_{1}^{f(m)} \uparrow \operatorname{supp}\left(c_{0} * c_{1}^{m} d_{1}^{n}\right) \quad \text { for all } m \in \omega \text { and } n \in \mathbf{Z} .
$$

Note that $a_{f}$ commutes with $a_{1}, b_{1}$ and $d_{1}$, and $a_{3} * c_{1}^{m} a_{f}=a_{0} * b_{1}^{f(m)} c_{1}^{m}$ for all $m \in \omega$. 
Finally, for technical reasons, we need one extra element $\hat{a} \in \mathbf{A}(\mathbf{R})$ defined by $\operatorname{supp}(\hat{a}) \subseteq \cup\left\{\operatorname{supp}\left(c_{0} * c_{1}^{m} d_{1}^{n} w\right): m, n \in \mathbf{Z}, w \in W\right\}$, and $\hat{a} \uparrow \operatorname{supp}\left(c_{0} * c_{1}^{m} d_{1}^{n} w\right)=$ $b_{1}^{m+1} \uparrow \operatorname{supp}\left(c_{0} * c_{1}^{m} d_{1}^{n} w\right)$ for all $m, n \in \mathbf{Z}, w \in W$. Note that $a_{s}=(\hat{a} \vee e) \wedge$ $a_{3}^{-1} d_{0} a_{3}, s$ being the successor function. Also $\alpha \hat{a} \geqslant \alpha$ for all $\alpha \in \operatorname{supp}\left(a_{6}\right)$; hence $a_{6} \wedge\left(\hat{a}^{-1} \vee e\right)=e$.

\section{The basic lattice-ordered group.}

In $\mathbf{A}(\mathbf{R})$ we constructed five "levels" of $\mathbf{Z}$. We now write down a finite presentation which will capture this information and will be a close approximation to the picture.

Let $G$ be the finitely-presented lattice-ordered group with generators the formal symbols $a_{0}, a_{1}, a_{2}, a_{3}, a_{4}, a_{5}, a_{6}$ and $a_{7}$ and relations

$$
\begin{array}{ll}
a_{0} \wedge a_{2}=e, & a_{1} \geqslant e, \\
a_{1}^{-1} a_{0} a_{1} \leqslant a_{2}, & a_{1}^{-1} a_{2} a_{1} \leqslant a_{2}, \\
a_{i} \leqslant b_{i}(i=0,1), & a_{1} b_{0}=b_{0} a_{1}, \\
a_{1} b_{1}=b_{1} a_{1}, & a_{1} c_{1}=c_{1} a_{1}, \\
a_{1} d_{1}=d_{1} a_{1}, & a_{3}^{-1} d_{0} a_{3} \wedge d_{1}\left(a_{3}^{-1} d_{0} a_{3}\right)^{-1}=e, \\
a_{0} a_{4}=a_{4} a_{0}, & a_{4}^{-1} a_{1} a_{4}=b_{1}, \\
a_{4}^{-1} b_{1} a_{4}=c_{1}, & a_{4}^{-1} c_{1} a_{4}=d_{1}, \\
a_{0} a_{5}=a_{5} a_{0}, & a_{5}^{-1} b_{0} a_{5}=c_{0}, \\
c_{1} a_{5}=a_{5} c_{1}, & a_{6} \wedge a_{7}=e, \\
c_{1}^{-1}\left(a_{6} \vee a_{7}\right) c_{1}=a_{6} \vee a_{7}, & a_{0} \wedge a_{6} a_{0}^{-1}=e, \\
c_{1}^{-1} a_{6} c_{1} \leqslant a_{6}, & c_{1} a_{7} c_{1}^{-1} \leqslant a_{7}, \\
c_{1} a_{0} c_{1}^{-1} \leqslant a_{7}, & a_{1} b_{0}^{-1} \wedge b_{0}=e
\end{array}
$$

where

$$
b_{i} \equiv a_{3}^{-1} a_{i} a_{3}, \quad c_{i} \equiv a_{3}^{-2} a_{i} a_{3}^{2}, \quad d_{i} \equiv a_{3}^{-3} a_{i} a_{3}^{3} \quad(i=0,1) .
$$

Observe that all of the above relations hold in $\mathbf{A}(\mathbf{R})$ under the natural interpretation.

Note that we write $x \leqslant y$ as a shorthand for $x \wedge y=x$.

We will continue to use $x * y$ for $y^{-1} x y$ and $x * y z$ for $x *(y z)$. Observe that $(x * y) * z=x * y z$.

LEMMA 1. In $G$, the following facts hold:

(i) $b_{1} c_{0}=c_{0} b_{1} ; c_{1} d_{0}=d_{1} c_{1}$.

(ii) $b_{1} c_{1}=c_{1} b_{1} ; c_{1} d_{1}=d_{1} c_{1} ; b_{1} d_{1}=d_{1} b_{1}$.

(iii) $a_{0} * a_{1}^{n} \leqslant a_{2}$ for all $n \in \mathbf{Z}^{+}$.

(iv) $\left(a_{0} * a_{1}^{n}\right) \wedge\left(a_{0} * a_{1}^{m}\right)=e$ unless $n=m$.

(v) $\left(b_{0} * b_{1}^{n}\right) \wedge\left(b_{0} * b_{1}^{m}\right)=e$ unless $n=m$. 
(vi) $\left(c_{0} * c_{1}^{n}\right) \wedge\left(c_{0} * c_{1}^{m}\right)=e$ unless $n=m$.

(vii) $\left(d_{0} * d_{1}^{n}\right) \wedge\left(d_{0} * d_{1}^{m}\right)=e$ unless $n=m$.

(viii) $\left(a_{0} * a_{1}^{m} b_{1}^{n} c_{1}^{k} d_{1}^{p}\right) \wedge\left(a_{0} * a_{1}^{m \prime} b_{1}^{n \prime} c_{1}^{k \prime} d_{1}^{p^{\prime}}\right)=e$ unless $\left(m=m^{\prime}, n=n^{\prime}, k=k^{\prime}\right.$ and $\left.p=p^{\prime}\right)$.

(ix) $a_{0} * c_{1}^{m} \leqslant a_{3}^{-1} d_{0} a_{3}$ for all $m \in \omega$.

(x) $a_{0} * c_{1}^{m} \leqslant a_{6}$ if and only if $m \in \omega$.

(xi) $a_{0} * a_{1}^{m} a_{5}=a_{0} * b_{1}^{m}$ for all $m \in \mathbf{Z}$.

(xii) $a_{1} \leqslant b_{1} \leqslant c_{1} \leqslant d_{1}$.

Proof. (i) and (ii) follow immediately by conjugating the relations $a_{1} b_{0}=b_{0} a_{1}$, $a_{1} b_{1}=b_{1} a_{1}$ and $a_{1} c_{1}=c_{1} a_{1}$ of $G$ by $a_{3}$ or $a_{3}^{2}$.

(iii) follows from the relations $a_{1}^{-1} a_{0} a_{1} \leqslant a_{2}$ and $a_{1}^{-1} a_{2} a_{1} \leqslant a_{2}$ by induction on $n$.

(iv) follows from (iii) and the relation $a_{0} \wedge a_{2}=e$.

(v), (vi) and (vii) now follow by conjugating (iv) by $a_{3}, a_{3}^{2}$ and $a_{3}^{3}$ respectively.

(viii) Since $a_{1}$ commutes with $b_{0}, a_{0} \leqslant b_{0}$ implies $a_{0} * a_{1}^{n} \leqslant b_{0}$ for all $n \in \mathbf{Z}$. Conjugating by $a_{3}$ and $a_{3}^{2}$ gives $b_{0} * b_{1}^{n} \leqslant c_{0}$ and $c_{0} * c_{1}^{n} \leqslant d_{0}$, respectively, for all $n \in \mathbf{Z}$. Hence $a_{0} * a_{1}^{m} b_{1}^{n} c_{1}^{k} \leqslant d_{0}$ and $a_{0} * a_{1}^{m^{\prime}} b_{1}^{n^{\prime}} c_{1}^{k^{\prime}} \leqslant d_{0}$. (viii) now follows by successively using (vii), (vi), (v) and (iv).

(ix) Note that $a_{0} * c_{1}^{m} \leqslant d_{0}$ for all $m \in \omega$ (as in (viii)). Since $a_{0} \leqslant b_{0}$,

$$
d_{0}=a_{0} * a_{3}^{3} \leqslant b_{0} * a_{3}^{3}=a_{3}^{-1} d_{0} a_{3} .
$$

Hence $a_{0} * c_{1}^{m} \leqslant a_{3}^{-1} d_{0} a_{3}$ for all $m \in \omega$.

(x) is similar to (iii) using the relations involving $a_{6}$ and $a_{7}$.

(xi) Since $a_{1} b_{0}^{-1} \wedge b_{0}=e, a_{1} \wedge b_{0}=b_{0}$ by Lemma 0(iii). But $a_{0} \leqslant b_{0}$; so $a_{0} * a_{1}^{m}$ $=a_{0} * b_{0}^{m}$ for all $m \in \mathbf{Z}$ by Lemma 0(ii). Thus $a_{0} * a_{1}^{m} a_{5}=a_{0} * b_{0}^{m} a_{5}=a_{0} * c_{0}^{m}$. But $b_{1} c_{0}^{-1} \wedge c_{0}=e$ (conjugate $a_{1} b_{0}^{-1} \wedge b_{0}=e$ by $a_{3}$ ); so $a_{0} * c_{0}^{m}=a_{0} * b_{1}^{m}$ by Lemma G(ii) again. Therefore $a_{0} * a_{1}^{m} a_{5}=a_{0} * b_{1}^{m}$ for all $m \in \mathbf{Z}$.

(xii) Since $a_{1} \leqslant b_{1}=a_{3}^{-1} a_{1} a_{3}, b_{1} \leqslant c_{1} \& c_{1} \leqslant d_{1}$ (conjugate by $a_{3} \& a_{3}^{2}$ ).

\section{Coding recursive functions into lattice-ordered groups.}

We write $(\mathbf{a} ; \mathbf{r}(\mathbf{a})=e)$ for the $G$ described above.

We observe that $G$ contains the five "levels" of $\mathbf{Z}$, as described above.

Our main goal in this section will be to code recursive functions (from $\omega$ into $\omega$ ) into finitely presented lattice-ordered groups.

A function $f \in{ }^{\omega} \omega$ is said to be representable if there are a finite number of generators $\mathbf{x}(f)$-including a and $a_{f}-$ and a finite number of words $\mathbf{s}(f)$ in these generators such that the relations $\mathbf{s}(f)=e$ hold in $\mathbf{A}(\mathbf{R})$ in the natural interpretation and, in $G(f)=(\mathbf{x}(f) ; \mathbf{r}(\mathbf{a})=e, \mathbf{s}(f)=e), a_{f}$ commutes with $a_{1}, b_{1}$ and $d_{1}$, and for all $m \in \omega$

$$
a_{0} * c_{1}^{m} a_{f}=a_{0} * b_{1}^{f(m)} c_{1}^{m} .
$$

By Lemma 1(viii), $a_{f}$ is well defined $\left(a_{0} * c_{1}^{m} \& a_{0} * f_{1}^{f(m)} c_{1}^{m}\right.$ are disjoint from $a_{0} * c_{1}^{n} \& a_{0} * b_{1}^{f(n)} c_{1}^{n}$ for all $\left.n \neq m\right)$. 
Note that in $\mathbf{A}(\mathbf{R}), a_{f}$ has all these properties. Indeed, all the relations that we will write down hold in $\mathbf{A}(\mathbf{R})$ in the natural interpretation as can be easily checked.

All generators of the finitely presented lattice-ordered groups we will construct will be of the form a, $\hat{a}$ or $a_{g}$ for some functions $g \in{ }^{\omega} \omega$.

Our main goal will be to prove that every recursive $f \in \epsilon^{\omega} \omega$ is representable.

To achieve this, it is easiest to take a classification of recursive functions of one variable given by Julia Robinson:

A function $f \in{ }^{\omega} \omega$ is obtained from $g, h, u, v \in{ }^{\omega} \omega$ by general recursion if (i) $f g=u$, (ii) $f h=v f$, and (iii) each $n \in \omega$ belongs to the range of one of the functions $h^{k} g(k \in \omega)$.

The first result from recursion theory that we will need is:

I. (Julia Robinson [10]) The class of recursive functions is the smallest class of numerical functions which is closed under composition and general recursion, and contains the zero function $\theta(\theta(n)=0)$ and the successor function $s(s(n)=n+1)$.

LEMMA 2. Every recursive function is representable.

Proof. Firstly, $\theta(n) \equiv 0$ is clearly representable-adjoin to $G$ the generator $a_{\theta}$ and the relation $a_{\theta}=e$.

Secondly, for $s$ the successor function, adjoin to $G$ the generators $\hat{a}$ and $a_{s}$, and the relations: $\hat{a}$ commutes with $a_{1}, b_{1}$ and $d_{1}$;

$$
\begin{gathered}
c_{1} \hat{a}=\hat{a} b_{1} c_{1} ; \quad a_{0} * \hat{a}=a_{0} * b_{1} ; \\
(\hat{a} \vee e)\left[(\hat{a} \vee e) \wedge a_{3}^{-1} d_{0} a_{3}\right]^{-1} \wedge a_{3}^{-1} d_{0} a_{3}=e ; \\
a_{s}=(\hat{a} \vee e) \wedge a_{3}^{-1} d_{0} a_{3} \text { and } a_{6} \wedge\left(\hat{a}^{-1} \vee e\right)=e .
\end{gathered}
$$

Since $\hat{a}$ commutes with $a_{1}, b_{1}$ and $d_{1}$, so does $\hat{a} \vee e$. Moreover, so does $a_{3}^{-1} d_{0} a_{3}$ by Lemmas 0(iii) and 1(xii) (with $x=a_{3}^{-1} d_{0} a_{3}, y=d_{1}$ and $z=a_{1}, b_{1}$ and $d_{1}$ ). Thus $a_{s}$ commutes with $a_{1}, b_{1}$ and $d_{1}$. We now prove $a_{0} * c_{1}^{m} \hat{a}=a_{0} * b_{1}^{s(m)} c_{1}^{m}$ for all $m \in \omega$ by induction.

For $m=0, a_{0} * c_{1}^{0} \hat{a}=a_{0} * \hat{a}=a_{0} * b_{1}=a_{0} * b_{1}^{s(0)} c_{1}^{0}$.

Assume $a_{0} * c_{1}^{m} \hat{a}=a_{0} * b_{1}^{s(m)} c_{1}^{m}$. Then

$$
\begin{aligned}
a_{0} * c_{1}^{m+1} \hat{a} & =a_{0} * c_{1}^{m} c_{1} \hat{a}=a_{0} * c_{1}^{m} \hat{a} b_{1} c_{1} \\
& =a_{0} * b_{1}^{s(m)} c_{1}^{m} b_{1} c_{1}=a_{0} * b_{1}^{s(m+1)} c_{1}^{m+1}
\end{aligned}
$$

as required.

Now $e=a_{3}^{-1} d_{0} a_{3} \wedge d_{1}\left(a_{3}^{-1} d_{0} a_{3}\right)^{-1}$, so $d_{1}=a_{3}^{-1} d_{0} a_{3} \vee d_{1}\left(a_{3}^{-1} d_{0} a_{3}\right)^{-1}$. Since $(\hat{a} \vee e)\left[(\hat{a} \vee e) \wedge a_{3}^{-1} d_{0} a_{3}\right]^{-1} \wedge a_{3}^{-1} d_{0} a_{3}=e$ and $a_{0} * c_{1}^{m} \leqslant a_{3}^{-1} d_{0} a_{3}$ (by Lemma (ix)), $a_{0} * c_{1}^{m}\left[(\hat{a} \vee e) \wedge a_{3}^{-1} d_{0} a_{3}\right]=a_{0} * c_{1}^{m}(\hat{a} \vee e)$ for all $m \in \omega$ by Lemma 0 (ii). Hence, by the above and the definition of $a_{s}\left(=(\hat{a} \vee e) \wedge a_{3}^{-1} d_{0} a_{3}\right)$,

$$
a_{0} * c_{1}^{m} a_{s}=a_{0} * c_{1}^{m}(\hat{a} \vee e) \text { for all } m \in \cdot \omega .
$$

But $a_{6} \wedge\left(\hat{a}^{-1} \vee e\right)=e$, so $a_{0} * c_{1}^{m} \wedge\left(\hat{a}^{-1} \vee e\right)=e(m \in \omega)$ by Lemma $1(\mathrm{x})$. By Lemma 0(i),

$$
a_{0} * c_{1}^{m}(e \vee \hat{a}) \hat{a}^{-1}=a_{0} * c_{1}^{m}\left(\hat{a}^{-1} \vee e\right)=a_{0} * c_{1}^{m}
$$


and hence

$$
a_{0} * c_{1}^{m}(e \vee \hat{a})=a_{0} * c_{1}^{m} \hat{a}=a_{0} * b_{1}^{s(m)} c_{1}^{m}
$$

for all $m \in \omega$. Therefore $a_{0} * c_{1}^{m} a_{s}=a_{0} * b_{1}^{s(m)} c_{1}^{m}$ for all $m \in \omega$. Thus $s$ is representable.

We next show that if $f$ and $g$ are representable, then so is $h=g f$. Let $G(h)$ have generators $\mathbf{x}(f) \cup \mathbf{x}(g) \cup\left\{a_{h}\right\}$, and relations: $\mathbf{s}(f)=e, \mathbf{s}(g)=e, a_{h}$ commutes with $a_{1}, b_{1}$ and $d_{1}$, and $a_{h}\left(a_{g} \uparrow a_{f}\right)^{-1} \wedge a_{6}=e$, where $x \dagger y$ is shorthand for $x * a_{4}^{-1} y^{-1} a_{5}$. Since $a_{0} * c_{1}^{m} \leqslant a_{6}$ for all $m \in \omega(\operatorname{Lemma} 1(\mathrm{x}))$ and $a_{h}\left(a_{g} \dagger a_{f}\right)^{-1} \wedge a_{6}$ $=e$,

$$
a_{0} * c_{1}^{m} a_{h}=a_{0} c_{1}^{m}\left(a_{g} \dagger a_{f}\right)
$$

by Lemma $0(\mathrm{i})$. So, in $G(h)$, using Lemma $1(\mathrm{xi})$,

$$
\begin{aligned}
a_{0} * c_{1}^{m} a_{h} & =a_{0} * c_{1}^{m}\left(a_{5}^{-1} a_{f} a_{4} a_{g} a_{4}^{-1} a_{f}^{-1} a_{5}\right) \\
& =a_{0} * b_{1}^{f(m)} c_{1}^{m} a_{4} a_{g} a_{4}^{-1} a_{f}^{-1} a_{5} \\
& =a_{0} * c_{1}^{f(m)} d_{1}^{m} a_{g} a_{4}^{-1} a_{f}^{-1} a_{5} \\
& =a_{0} * b_{1}^{g f(m)} c_{1}^{f(m)} d_{1}^{m} a_{4}^{-1} a_{f}^{-1} a_{5} \\
& =a_{0} * a_{1}^{h(m)} b_{1}^{f(m)} c_{1}^{m} a_{f}^{-1} a_{5} \\
& =a_{0} * a_{1}^{h(m)} c_{1}^{m} a_{5}=a_{0} * b_{1}^{h(m)} c_{1}^{m} .
\end{aligned}
$$

Hence the composition of representable functions is representable.

Lastly, we must show that if $g, h, u$ and $v$ are representable and $f$ is obtained from them by general recursion, then $f$ is representable. So assume the hypothesis and let $G(f)$ have a generators $a_{f}$ together with those used to show the representability of $g, h, u$ and $v$, and relations those required for $g, h, u$ and $v$ together with: $a_{f}$ commutes with $a_{1}, b_{1}$ and $d_{1}$;

$$
a_{u}\left(a_{f} \dagger a_{g}\right)^{-1} \wedge a_{6}=e \text { and }\left(a_{f} \dagger a_{h}\right)\left(a_{v} \dagger a_{f}\right)^{-1} \wedge a_{6}=e .
$$

Now each $m \in \omega$ belongs to the range of some $h^{k} g$. We prove, by induction on $k$, that for each $m \in \operatorname{range}\left(h^{k} g\right), a_{0} * c_{1}^{m} a_{f}=a_{0} * b_{1}^{f(m)} c_{1}^{m}$.

For $k=0$, let $m=g(n)$, say.

Thus $a_{0} * c_{1}^{n} a_{u}=a_{0} * b_{1}^{u(n)} c_{1}^{n}=a_{0} * b_{1}^{f(m)} c_{1}^{n}$ since $u$ is representable and $u=f g$. Since $a_{u}\left(a_{f} \dagger a_{g}\right)^{-1} \wedge a_{6}=e$, it follows that

$$
a_{0} * c_{1}^{n} a_{u}=a_{0} * c_{1}^{n}\left(a_{f} * a_{4}^{-1} a_{g}^{-1} a_{5}\right) .
$$

Hence

$$
a_{0} * b_{1}^{f(m)} c_{1}^{n} a_{5}^{-1} a_{g} a_{4}=a_{0} * c_{1}^{n} a_{5}^{-1} a_{g} a_{4} a_{f} .
$$

So

$$
a_{0} * a_{1}^{f(m)} c_{1}^{n} a_{g} a_{4}=a_{0} * b_{1}^{g(n)} c_{1}^{n} a_{4} a_{f} .
$$

Therefore

$$
a_{0} * b_{1}^{f(m)} c_{1}^{m} d_{1}^{n}=a_{0} * c_{1}^{m} d_{1}^{n} a_{f} .
$$

Since $d_{1} a_{f}=a_{f} d_{1}, a_{0} * b_{1}^{f(m)} c_{1}^{m}=a_{0} * c_{1}^{m} a_{f}$. 
Now assume that if $n \in \operatorname{range}\left(h^{k} g\right)$, then $a_{0} * c_{1}^{n} a_{f}=a_{0} * b_{1}^{f(n)} c_{1}^{n}$ and suppose $m \in \operatorname{range}\left(h^{k+1} g\right)$. So $m=h(n)$ for some $n \in \operatorname{range}\left(h^{k} g\right)$.

But

$$
a_{0} * c_{1}^{n}\left(a_{f} \dagger a_{h}\right)=a_{0} * c_{1}^{n}\left(a_{v} \dagger a_{f}\right)
$$

so

$$
a_{0} * c_{1}^{n} a_{5}^{-1} a_{h} a_{4} a_{f} a_{4}^{-1} a_{h}^{-1} a_{5}=a_{0} * c_{1}^{n} a_{5}^{-1} a_{f} a_{4} a_{v} a_{4}^{-1} a_{f}^{-1} a_{5} .
$$

By the hypotheses,

$$
a_{0} * c_{1}^{h(n)} d_{1}^{n} a_{f} a_{4}^{-1} a_{h}^{-1}=a_{0} * c_{1}^{f(n)} d_{1}^{n} a_{v} a_{4}^{-1} a_{f}^{-1} .
$$

Thus

$$
a_{0} * c_{1}^{m} a_{f} d_{1}^{n} a_{4}^{-1} a_{h}^{-1}=a_{0} * b_{1}^{v f(n)} c_{1}^{f(n)} d_{1}^{n} a_{4}^{-1} a_{f}^{-1}
$$

Hence

$$
a_{0} * c_{1}^{m} a_{f} d_{1}^{n}=a_{0} * b_{1}^{v f(n)} c_{1}^{f(n)} d_{1}^{n} a_{4}^{-1} a_{f}^{-1} a_{h} a_{4} .
$$

Since $v f(n)=f h(n)=f(m)$ and $a_{1}$ commutes with $b_{1}, c_{1}$ and $a_{f}$,

$$
a_{0} * c_{1}^{m} a_{f}=a_{0} * b_{1}^{f(n)} c_{1}^{n} a_{f}^{-1} a_{1}^{f(m)} a_{h} a_{4} d_{1}^{-n} \text {. }
$$

Therefore

$$
a_{0} * c_{1}^{m} a_{f}=a_{0} * c_{1}^{n} a_{h} a_{1}^{f(m)} a_{4} d_{1}^{-n}
$$

So

$$
a_{0} * c_{1}^{m} a_{f}=a_{0} * b_{1}^{h(n)} c_{1}^{n} a_{1}^{f(m)} a_{4} d_{1}^{-n} .
$$

As $h(n)=m$ and $b_{1}, c_{1}$ and $d_{1}$ commute,

$$
a_{0} * c_{1}^{m} a_{f}=a_{0} * b_{1}^{f(m)} c_{1}^{m} .
$$

By I, every recursive function is representable, and the proof of the lemma complete.

5. The proofs of the Theorem and Corollary. We now apply the results of $\S \S 2$ and 4 to prove the Theorem. We need the following fact:

II. There is a recursive function whose range is not a recursive set.

Let $f$ be a recursive function whose range $X$ is not recursive. Let $h$ be the characteristic function of $\omega \backslash X$; i.e.,

$$
h(m)= \begin{cases}0 & \text { if } m \in X, \\ 1 & \text { if } m \notin X .\end{cases}
$$

Note that $h$ is not recursive and so is not necessarily representable.

By Lemma 2, $f$ is representable. Adjoin to $G(f)$ a new generator $a_{h}$ and relations: $a_{h}$ commutes with $a_{1}, b_{1}$ and $d_{1}$, and $\left(a_{h}^{\dagger} a_{f}\right) \wedge a_{6}=e$. Let $H_{X}$ be the resulting finitely presented lattice-ordered group. The set of generators corresponds to the set $X_{0}$ mentioned in the introduction; the relations to $S_{0}$ mentioned there. Now since $a_{0} * c_{1}^{n} \leqslant a_{6}$ for all $n \in \omega$,

$$
a_{0} * c_{1}^{n}\left(a_{h} \dagger a_{f}\right)=a_{0} * c_{1}^{n}
$$


by Lemma $0(\mathbf{i})$. So

$$
a_{0} * c_{1}^{n} a_{5}^{-1} a_{f} a_{4} a_{h}=a_{0} * c_{1}^{n} a_{5}^{-1} a_{f} a_{4}
$$

Thus

$$
a_{0} * c_{1}^{f(n)} a_{h}=a_{0} * c_{1}^{f(n)} .
$$

This set of relations corresponds to the nonrecursive set $S$ of the introduction, whereas $R$ corresponds to the set $a_{0} * c_{1}^{m} a_{h}=a_{0} * c_{1}^{m}(m \in \omega)$.

However (by $\S 2$ ), in $\mathbf{A}(\mathbf{R})$, if $m \notin X$,

$$
a_{0} * c_{1}^{m} a_{h}=a_{0} * b_{1} c_{1}^{m} \neq a_{0} * c_{1}^{m} .
$$

Since all the defining relations of $H_{X}$ hold in $\mathbf{A}(\mathbf{R})$ in the given interpretation, in $H_{X}$,

$$
a_{0} * c_{1}^{m} a_{h}=a_{0} * c_{1}^{m} \quad \text { if and only if } m \in X .
$$

Consequently, we have achieved our goal and the finitely presented lattice-ordered group $H_{X}$ has insoluble word problem. Q.E.D.

Note that the word problem for $H_{X}$ has Turing degree at least that of $X$.

We now prove the Corollary.

If $L$ is any lattice-ordered group and $x \in L$, then $|x|=x \vee x^{-1} \geqslant e$; indeed $|x|=e$ if and only if $x=e\left[2\right.$, Lemma 1.11.4]. Hence $w_{1}, \ldots, w_{n}$ are simultaneously equal to $e$ if and only if $\left|w_{1}\right| \vee \cdots \vee\left|w_{n}\right|=e$. So any finite set of relations in any lattice-ordered group is equivalent to a single relation. The Corollary therefore follows immediately from the Theorem.

Appendix. In $\S 2$ we asserted the existence of certain elements of $\mathbf{A}(\mathbf{R})$. We did not wish to elaborate on the reasons for their existence earlier as we felt that a better understanding of our ideas would result if we postponed these technicalities. However, we must now prove the existence of $a_{3}, a_{4}, a_{5} \in \mathbf{A}(\mathbf{R})$. The proof is essentially in [2, \$2.2]; its root is [4].

Choose $\alpha_{0} \in \operatorname{supp}\left(a_{0}\right)$. Let $\beta_{0}=\inf \left(\operatorname{supp}\left(a_{0}\right)\right)$ and $\gamma_{0}=\inf \left(\operatorname{supp}\left(b_{0}\right)\right)$. Observe that $\sup \left(\operatorname{supp}\left(a_{0}\right)\right)<\beta_{0} b_{0}$ and $\sup \left(\operatorname{supp}\left(b_{0}\right)\right)<\gamma_{0} c_{0}$ by $\S 2(\mathrm{c})$ and (d) respectively. Let $f_{0}$ be an order-preserving one-to-one map of $\left[\alpha_{0}, \alpha_{0} a_{0}\right]$ onto $\left[\alpha_{0}, \alpha_{0} b_{0}\right]$. Let $f_{n}=a_{0}^{-n} f_{0} b_{0}^{n}$, an order-preserving one-to-one map of $\left[\alpha_{0} a_{0}^{n}, \alpha_{0} a_{0}^{n+1}\right]$ onto $\left[\alpha_{0} b_{0}^{n}, \alpha_{0} b_{0}^{n+1}\right](n \in \mathbf{Z})$. Define $f: \operatorname{supp}\left(a_{0}\right) \rightarrow \operatorname{supp}\left(b_{0}\right)$ by $f \uparrow\left[\alpha_{0} a_{0}^{n}, \alpha_{0} a_{0}^{n+1}\right]=f_{n}$ $\left(n \in \mathbf{Z}\right.$ ); i.e. $f=\cup\left\{f_{n}: n \in \mathbf{Z}\right\}$. We can extend $f$ to an order-preserving one-to-one map $g_{0}$ of $\left[\beta_{0}, \beta_{0} b_{0}\right]$ onto $\left[\gamma_{0}, \gamma_{0} c_{0}\right]$. Let $g_{n}=b_{0}^{-n} g_{0} c_{0}^{n}:\left[\beta_{0} b_{0}^{n}, \beta_{0} b_{0}^{n+1}\right] \rightarrow$ $\left[\gamma_{0} c_{0}^{n}, \gamma_{0} c_{0}^{n+1}\right](n \in \mathbf{Z})$ and $g=\bigcup\left\{g_{n}: n \in \mathbf{Z}\right\}$. So $g: \operatorname{supp}\left(b_{0}\right) \rightarrow \operatorname{supp}\left(c_{0}\right)$. We can extend $g$ to an element of $\mathbf{A}(\mathbf{R})$, say $a_{3}$. Since $a_{3}$ extends both $f$ and $g$, it follows by an easy computation that $a_{3}^{-1} a_{0} a_{3}=b_{0}$ and $a_{3}^{-1} b_{0} a_{3}=c_{0} . \quad\left(\operatorname{supp}\left(a_{3}^{-1} a_{0} a_{3}\right)=\right.$ $\operatorname{supp}\left(a_{0}\right) a_{3}=\operatorname{supp}\left(a_{0}\right) f=\operatorname{supp}\left(b_{0}\right), \quad$ and if $\sigma \in\left[\alpha_{0} b_{0}^{n}, \alpha_{0} b_{0}^{n+1}\right], \quad \sigma a_{3}^{-1} a_{0} a_{3}=$ $\sigma f_{n}^{-1} a_{0} f_{n+1}=\sigma b_{0}$. The other equality is proved similarly.) This shows that $a_{3}$ exists.

The constructions of $a_{4}$ and $a_{5}$ are similar. Let $\delta_{0}=\inf \left(\operatorname{supp}\left(c_{0}\right)\right)$. Note that $\sup \left(\operatorname{supp}\left(c_{0}\right)\right)<\boldsymbol{\delta}_{0} d_{0}$. Let $f_{0}$ be a one-to-one order-preserving map of $\left[\beta_{0}, \beta_{0} b_{0}\right]$ onto $\left[\beta_{0}, \beta_{0} c_{0}\right]$ such that $f_{0} \uparrow \operatorname{supp}\left(a_{0}\right)=e$. Let $f_{n}=b_{0}^{-n} f_{0} c_{0}^{n}(n \in \mathbf{Z})$, and $f: \operatorname{supp}\left(b_{0}\right)$ $\rightarrow \operatorname{supp}\left(c_{0}\right)$ be given by $f=\bigcup\left\{f_{n}: n \in \mathbf{Z}\right\}$. Let $g_{0}:\left[\gamma_{0}, \gamma_{0} c_{0}\right] \rightarrow\left[\delta_{0}, \delta_{0} d_{0}\right]$ be a 
one-to-one order-preserving onto map extending $f$. Let $g_{n}=c_{0}^{-n} g_{0} d_{0}^{n}(n \in \mathbf{Z})$ and $g$ : $\operatorname{supp}\left(c_{0}\right) \rightarrow \operatorname{supp}\left(d_{0}\right)$ be defined by $g=\cup\left\{g_{n}: n \in \mathbf{Z}\right\}$. We continue this extension process to

$$
\operatorname{supp}\left(d_{0}\right) \rightarrow \operatorname{supp}\left(x_{0}\right), \operatorname{supp}\left(x_{0}\right) \rightarrow \operatorname{supp}\left(y_{1}\right), \ldots, \operatorname{supp}\left(y_{n}\right) \rightarrow \operatorname{supp}\left(y_{n+1}\right), \ldots
$$

to obtain $h$, an order-preserving permutation of the interval $\cup\left\{\operatorname{supp}\left(y_{n}\right): n \in \mathbf{Z}^{+}\right\}$. By construction, $h^{-1} a_{0} h=a_{0}, h^{-1} a_{1} h=b_{1}, h^{-1} b_{1} h=c_{1}$ and $h^{-1} c_{1} h=d_{1}$. Extend $h$ to an element of $\mathbf{A}(\mathbf{R})$, call it $a_{4}$. (Of course, if $\mathbf{R}=\bigcup\left\{\operatorname{supp}\left(y_{n}\right): n \in \mathbf{Z}^{+}\right\}$, this step is unnecessary.) Then $a_{4} \in \mathbf{A}(\mathbf{R})$ has all the desired properties.

For $a_{5}$, let $f$ be defined as for $a_{4}$. Let $g_{0}:\left[\delta_{0}, \delta_{0} d_{0}\right] \rightarrow\left[\delta_{0}, \delta_{0} d_{0}\right]$ be an order-preserving one-to-one onto map extending $f$. Let $g_{n}=d_{0}^{-n} g_{0} d_{0}^{n}(n \in \mathbf{Z})$ and $g$ be the order-preserving permutation of $\operatorname{supp}\left(d_{0}\right)$ given by $\cup\left\{g_{n}: n \in \mathbf{Z}\right\}$. Then $g^{-1} d_{0} g=$ $d_{0}$. Since $g$ extends $f, g^{-1} a_{0} g=a_{0}$ and $g^{-1} b_{0} g=c_{0}$. Let $h_{n, w}$ be that order-preserving permutation of $\operatorname{supp}\left(d_{0} * d_{1}^{n} w\right)$ defined by $g * d_{1}^{n} w(n \in \mathbf{Z}, w \in W)$. Then $h=\bigcup\left\{h_{n, w}: n \in \mathbf{Z}, w \in W\right\}$ has the properties $h^{-1} a_{0} h=a_{0}, h^{-1} b_{0} h=c_{0}$ and $h^{-1} c_{1} h=c_{1}$. Any extension of $h$ to an element of $\mathbf{A}(\mathbf{R})$ can be taken as $a_{5}$.

\section{REFERENCES}

0. W. W. Boone, The word problem, Ann. of Math. (2) 70 (1959), 207-265.

1. J. L. Britton, The word problem, Ann. of Math. (2) 77 (1963), 16-32.

2. A. M. W. Glass, Ordered permutation groups, London Math. Soc. Lecture Notes Ser., No. 55 , Cambridge Univ. Press, London and New York, 1981.

3. _ The word problem for lattice-ordered groups, Proc. Edinburgh Math. Soc. (2) 19 (1975), 217-219.

4. W. Charles Holland, The lattice-ordered group of automorphisms of an ordered set, Michigan Math. J. 10 (1963), 399-408.

5. W. C. Holland and S. H. McCleary, Solvability of the word problem in free lattice-ordered groups, Houston J. Math. 5 (1979), 99-105.

6. N. G. Khisamiev, Universal theory of lattice-ordered abelian groups, Algebra i Logika 5 (1966), 71-76. (Russian)

7. W. Magnus, Das Identitätsproblem für Gruppen mit einer definierenden Relation, Math. Ann. 106 (1932), 295-307.

8. S. H. McCleary, A solution of the word problem in free normal valued lattice-ordered groups, Ordered Groups (J. E. Smith, G. O. Kenny and R. N. Ball, eds.) (Proc. Boise State Conf., 1978), Marcel Dekker, New York, 1980, pp. 107-129.

9. Ralph McKenzie and Richard J. Thompson, An elementary construction of unsolvable word problems in group theory, Word Problems: Decision Problems and the Burnside Problem in Group Theory (W. W. Boone, F. B. Cannonito and R. C. Lyndon, eds.) North-Holland, Amsterdam, 1973, pp. 457-478.

10. Julia Robinson, Recursive functions of one variable, Proc. Amer. Math. Soc. 19 (1968), 815-820.

Department of Mathematics, Bowling Green State University, Bowling Green, Ohio 43403 (Current address of A. M. W. Glass)

Department of Mathematics, Ben-Gurion University of The Negev, BeERSheva, IsRael

Current address (Yuri Gurevich): Department of Computer Science, University of Michigan, Ann Arbor, Michigan 48109 